\title{
Long non-coding RNA CUDR promotes malignant phenotypes in pancreatic ductal adenocarcinoma via activating AKT and ERK signaling pathways
}

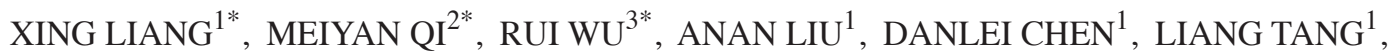 \\ JUN CHEN $^{1}$, XIANGUI HU ${ }^{4}$, WEI $\mathrm{LI}^{5}$, LIXING ZHAN ${ }^{2}$ and CHENGHAO SHAO ${ }^{1,4}$ \\ ${ }^{1}$ Department of Pancreatic-Biliary Surgery, Changzheng Hospital, Second Military Medical University, Shanghai 200003; \\ ${ }^{2}$ Key Laboratory of Food Safety Research, Institute for Nutritional Sciences, Shanghai Institutes for Biological Sciences, \\ Chinese Academy of Sciences, University of The Chinese Academy of Sciences, Shanghai 200031; \\ ${ }^{3}$ The First Department of Biliary Surgery, Eastern Hepatobiliary Surgery Hospital; ${ }^{4}$ Department of Pancreatic Surgery, \\ Changhai Hospital, Second Military Medical University, Shanghai 200433; ${ }^{5}$ General Surgical Department, \\ Sir Run Run Shaw Hospital affiliated with The Zhejiang University School of Medicine,
}

Hangzhou, Zhejiang 310016, P.R. China

Received May 29, 2018; Accepted August 10, 2018

DOI: $10.3892 /$ ijo.2018.4574

\begin{abstract}
Pancreatic ductal adenocarcinoma (PDAC) is one of the most lethal malignancies, with a marked potential for invasion and metastasis. Emerging evidence has suggested that dysregulation of long non-coding RNAs (lncRNAs) is associated with the development of multiple types of cancer. However, the function of lncRNAs in PDAC is poorly known. In the present study, a microarray assay was used to screen for differently expressed IncRNAs in PDAC and it was identified that cancer upregulated drug resistance (CUDR) was upregulated in PDAC. CUDR increased PDAC cell proliferation, migration and invasion, inhibited apoptosis, and promoted drug resistance; it also regulated the PDAC cell epithelial-mesenchymal transition. The CUDR-induced PDAC malignant phenotypes is via the protein kinase $\mathrm{B}$ and extracellular-signal-regulated kinase signaling pathways. Downregulation of CUDR may be
\end{abstract}

Correspondence to: Professor Chenghao Shao, Department of Pancreatic-Biliary Surgery, Changzheng Hospital, Second Military Medical University, Fengyang Road 415, Shanghai 200003, P.R. China E-mail: shaochenghao_czyy@163.com

Professor Lixing Zhan, Key Laboratory of Food Safety Research, Institute for Nutritional Sciences, Shanghai Institutes for Biological Sciences, Chinese Academy of Sciences, University of the Chinese Academy of Sciences, Taiyuan Road 294, Shanghai 200031, P.R. China E-mail: 1xzhan@sibs.ac.cn

*Contributed equally

Key words: pancreatic neoplasm, long non-coding RNA cancer upregulated drug resistance, epithelial-mesenchymal transition, protein kinase B, extracellular-signal-regulated kinase a novel therapeutic strategy to prevent PDAC development and drug resistance in the future.

\section{Introduction}

Pancreatic ductal adenocarcinoma (PDAC) is one of the most aggressive human digestive malignancies and ranks fourth as the cause of cancer-associated mortality in the USA (1). In total, $90 \%$ of patients with PDAC are in an advanced stage at diagnosis, and $\sim 80 \%$ are not eligible for radical resection because of tumor invasion of the adjacent organs or extensive metastasis (2). The remaining patients have a poor prognosis regardless of surgical status; their 5-year survival rate is $<44 \%$, and their mortality rate is $\sim 100 \%(3,4)$. Therefore, an increased understanding of the molecular mechanisms that mediate PDAC proliferation, invasion and metastasis is urgently required.

Increasing evidence has suggested that long non-coding RNAs (lncRNAs) serve multiple functions in tumor initiation and development, and represent novel targets for cancer diagnosis and therapies $(5,6)$. Several lncRNAs have been associated with pancreatic cancer and may be used as biomarkers of PDAC (7-9).

In the present study, a microarray assay was used to screen for differently expressed lncRNAs in PDAC. Furthermore, the expression of the lncRNA cancer upregulated drug resistance (CUDR) was identified in PDAC, and its biological function and underlying molecular mechanism were investigated in PDAC cells.

\section{Materials and methods}

Tissue samples. A total of 25 pairs of tumor tissue samples and matched paracarcinoma tissue samples were obtained from patients with PDAC (15 males and 10 females; average age, 
58.3 \pm 10.4 years; range, 35-76 years) between February 2012 and August 2013 from Changhai Hospital, Second Military Medical University (Shanghai, China). All patients or their relatives provided written informed consent for their tissues to be used for scientific research, and the present study was approved by the Committee on Ethics of Biomedicine, Second Military Medical University. None of the patients had received radiotherapy, chemotherapy or other antitumor therapy prior to surgery. All diagnoses were confirmed by two certified pathologists on the basis of pathological and/or cytological evidence. All specimens were immediately frozen with RNA-stabilizing agent (RNAlater ${ }^{\circledR}$; Qiagen $\mathrm{GmbH}$, Hilden, Germany) in liquid nitrogen at the time of surgery, and stored at $-80^{\circ} \mathrm{C}$ until required.

RNA extraction and reverse transcription-quantitative polymerase chain reaction (RT- $q P C R)$. Total RNA was extracted from tissues or cells using TRIzol ${ }^{\circledR}$ reagent (Invitrogen; Thermo Fisher Scientific, Inc.), according to the manufacturer's protocol. RNA was reverse-transcribed into cDNA using a PrimeScript RT-PCR kit (Takara Biotechnology Co., Ltd., Dalian, China), according to the manufacturer's protocol. The relative expression level of the targets was determined by qPCR using SYBR Premix Ex Taq (Takara Biotechnology Co., Ltd. Thermocycling conditions: Following an initial denaturation for $30 \mathrm{sec}$ at $95^{\circ} \mathrm{C}, 40$ cycles of $5 \mathrm{sec}$ at $95^{\circ} \mathrm{C}$ and $1 \mathrm{~min}$ at $60^{\circ} \mathrm{C}$ were performed, followed by a dissociation stage for $15 \mathrm{sec}$ at $95^{\circ} \mathrm{C}, 1 \mathrm{~min}$ at $60^{\circ} \mathrm{C}$ and $15 \mathrm{sec}$ at $95^{\circ} \mathrm{C}$ ). The expression of CUDR in clinical samples was normalized to that of GAPDH. The relative level of CUDR expression in PDAC tissues (T) was compared with that in adjacent normal tissues $(\mathrm{N})$, and the fold change of the target lncRNAs was calculated using the $2^{-\Delta \Delta C q}$ method and was $\log _{2}$-transformed $\left[\log _{2}(\mathrm{~T} / \mathrm{N})\right](10)$. Primers for RT-qPCR are presented in Table I. RT-qPCR for each sample was performed in triplicate.

LncRNA microarrays and analysis. Frozen PDAC tissues and matched adjacent non-tumor samples were sent to KangChen Biotechnology Co. Ltd. (Shanghai, China) for IncRNA extraction, quantity testing, lncRNA labeling and analysis. The Arraystar Human LncRNA Array version 2.0 was designed for profiling lncRNAs and protein-coding RNAs in the human genome. A total of 33,045 lncRNAs were collected from authoritative data sources including RefSeq (www.ncbi.nlm. nih.gov/refseq), University of California Santa Cruz Known Genes (www.genomaize.org), Ensembl (ensemblgenomes.org) and a number of associated references (11-14). A paired t-test was used to compare mean differences when the observations had been obtained in pairs. Hierarchical clustering analysis was performed using Cluster (version 3.0) and Java TreeView software (version 1.1.4r3) (both from Stanford University, Stanford, CA, USA). All the microarray data were published in the Gene Expression Omnibus database (www.ncbi.nlm.nih. gov/geo) under accession number GSE101094.

Cell culture and transfection. Human pancreatic cancer cell lines (Panc-1, CFPAC-1, Capan-1, Aspc-1, SW1990, BxPC-3, Miapaca-2 and HPAC) were purchased from the American Type Culture Collection (Manassas, VA, USA). Cells were maintained in RPMI-1640 medium or Dulbecco's modified
Eagle's medium (both from Gibco; Thermo Fisher Scientific, Inc.) supplemented with $10 \%$ fetal bovine serum (FBS; HyClone; GE Healthcare, Logan, UT, USA) and $1 \%$ penicillin/streptomycin (Invitrogen; Thermo Fisher Scientific,Inc.). HPDE cells (provided by Professor Lixing Zhan, University of The Chinese Academy of Sciences, Shanghai, China) were cultured in a keratinocyte serum-free medium supplemented with $5 \mathrm{ng} / \mathrm{ml}$ epidermal growth factor (Invitrogen; Thermo Fisher Scientific, Inc.) and $50 \mathrm{mg} / \mathrm{ml}$ bovine pituitary extract (Gibco; Thermo Fisher Scientific, Inc.). All cell lines were cultured in a $37^{\circ} \mathrm{C}$ incubator with $5 \% \mathrm{CO}_{2}$. Plasmids and siRNAs were transfected into cells using FuGENE ${ }^{\circledR}$ HD transfection reagent (Roche Diagnostics GmbH, Mannheim, Germany), according to the manufacturer's protocol. In order to inhibit the AKT or ERK activation, cells were treated with a specific AKT-activation inhibitor MK2206 $(2 \mu \mathrm{M})$ for $24 \mathrm{~h}$, or with a specific ERK-activation inhibitor PD98059 $(10 \mu \mathrm{M})$ for $24 \mathrm{~h}$ before performing MTT, flow cytometric analysis, would-healing, Transwell or western blot assays.

Stable cell line construction. The full-length cDNA of CUDR was synthesized, digested with BamHI and EcoRI restriction enzymes, cloned into pBABE-puro plasmid (provided by Professor Lixing Zhan, University of The Chinese Academy of Sciences, Shanghai, China), and were validated by sequencing (Biosune Biotechnology Co. Ltd., Shanghai, China). RNA interference (RNAi) sequences were designed using the short hairpin (sh)RNA library of RNAi Codex (codex.cshl.edu). They were synthesized and cloned into the plko.1-puro retroviral expression vector (Addgene, Inc., Cambridge, MA, USA) using flanking EcoRI and AgeI restriction sites. The sequences are presented in Table II.

The stable CUDR-overexpressing Panc-1 cell line (Panc-1CUDR) and the control Panc-1 cell line (Panc-1-Control) were generated using retroviral vectors. Retrovirus was produced by transiently transfecting 293-GPG packaging cells seeded in $10-\mathrm{cm}$ dishes with $10 \mu \mathrm{g}$ pBABE-puro-CUDR vector or empty pBABE-puro vector. At 2 days after transfection, viral supernatant was collected, filtered and added to the Panc-1 cells. After 2 days, cells were selected with $2 \mathrm{mg} / \mathrm{ml}$ puromycin for 2 weeks. Stable CUDR-knockdown CFPAC-1 cell lines (CFPAC-1-shCUDR-1 and CFPAC-1-shCUDR-2) and the control cell line (CFPAC-1-shControl) were generated using lentiviral vectors. Lentivirus was produced by transiently transfecting 293T packaging cells seeded in 6-cm dishes with $2.7 \mu \mathrm{g}$ packaging construct, $0.9 \mu \mathrm{g}$ construct expressing vesicular stomatitis virus glycoprotein and $2.7 \mu \mathrm{g}$ plko.1-puroshCUDR-1, plko.1-puro-shCUDR-2 or empty plko.1-puro vectors, respectively. The media from the transfected cells were collected as the cells were re-fed each day for 3 or 4 days. The virus-containing media were pooled, filtered and added to the CFPAC-1 cells. After 2 days, cells were selected with $2 \mathrm{mg} / \mathrm{ml}$ puromycin for 2 weeks.

MTT proliferation assay. Cells were seeded in 96-well plates at a density of 2,000 cells/well in triplicate and incubated overnight. MTT solution (20 $\mu \mathrm{l}$ at $5 \mathrm{mg} / \mathrm{ml}$; Sigma; Thermo Fisher Scientific, Inc.) was added to each well and plates were incubated for $4 \mathrm{~h}$ at $37^{\circ} \mathrm{C}$. The supernatant was aspirated and the formazan crystals that had formed were dissolved by the 
Table I. Primer sequences used.

\begin{tabular}{lll}
\hline Gene & \multicolumn{1}{c}{ Forward primer } & \multicolumn{1}{c}{ Reverse primer } \\
\hline CUDR & 5'-CCCCAACTGATCAATCAACC-3' & 5'-TGGAGAGATGATGGGACTCA-3' \\
E-cadherin & 5'-CTGCTGCAGGTCTCCTCTTG-3' & 5'-TGTCGACCGGTGCAATCTTC-3' \\
N-cadherin & 5'-GACAATGCCCCTCAAGTGTT-3' & 5'-CCATTAAGCCGAGTGATGGT-3' \\
Vimentin & 5'-AAGGCGAGGAGAGCAGGATT-3' & 5'-GGTCATCGTGATGCTGAGAAG-3' \\
Slug & 5'-TGATGAAGAGGAAAGACTACAG-3' & 5'-GCTCACATATTCCTTGTCACAG-3' \\
ZEB1 & 5'-TGCACTGAGTGTGGAAAAGC-3' & 5'-TGGTGATGCTGAAAGAGACG-3' \\
GAPDH & 5'-TGTTCGACAGTCAGCCGCATCTTC-3' & 5'-CATGTAGTTGAGGTCAATGAAGG-3'
\end{tabular}

CUDR, cancer upregulated drug resistance; E-cadherin, epithelial cadherin; N-cadherin, neuronal cadherin; ZEB1, zinc finger E-box-binding homeobox 1 .

Table II. RNA interference sequences of CUDR.

\begin{tabular}{lll}
\hline shRNA symbol & \multicolumn{1}{c}{ Forward } & \multicolumn{1}{c}{ Reverse } \\
\hline shCUDR-1 & 5'-CCGGGGTAATGTATCATCGGCTTAGCTCGA & 5'-AATTCAAAAAGGTAATGTATCATCGGCTT \\
& GCTAAGCCGATGATACATTACCTTTTTG-3' & AGCTCGAGCTAAGCCGATGATACATTACC-3' \\
shCUDR-2 & 5'-CCGGGGAATACTATTCGTATGATGGCTC & 5'-AATTCAAAAAGGAATACTATTCGTATGAT \\
& GAGCCATCATACGAATAGTATTCCTTTTTG-3' & GGCTCGAGCCATCATACGAATAGTATTCC-3' \\
\end{tabular}

CUDR, cancer upregulated drug resistance; shRNA/sh, short hairpin RNA.

addition of $150 \mu 1$ dimethylsulfoxide. The absorbance was measured at $490 \mathrm{~nm}$ in a Biotek Elx800 microplate reader (Molecular Devices, LLC, Sunnyvale, CA, USA). The mean values of 6 wells were calculated to determine cell proliferation every $24 \mathrm{~h}$.

Wound-healing assay. PDAC cells were cultured until reaching $80 \%$ confluence. Scratches were made by a 96 wounding replicator (V\&P Scientific, Inc., San Diego, CA, USA), and the medium was replaced with fresh RPMI-1640 medium containing $0.5 \%$ FBS. The cells were washed twice with PBS to remove the floating cells and were incubated with fresh RPMI-1640 medium containing 0.5\% FBS. Images of the scratches were captured at 0,8 and $24 \mathrm{~h}$ under a fluorescence microscope (1X71; Olympus Corporation, Tokyo, Japan) and wound-healing rates were determined.

Transwell migration and invasion assays. For the Transwell migration assay, PDAC cells were collected with serumfree medium and seeded into the upper chamber of 24-well Transwell dishes (Corning Incorporated, Corning, NY, USA) at an initial concentration of $4 \times 10^{4}$ cells/well, and the lower chamber was filled with culture medium containing 10\% FBS as the chemoattractant. Following incubation at $37^{\circ} \mathrm{C}$ for $24 \mathrm{~h}$, non-migrating cells were removed with a cotton swab, and the remaining cells were fixed, stained with crystal violet at $37^{\circ} \mathrm{C}$ for $10 \mathrm{~min}$ and images were captured under a fluorescence microscope (1X71; Olympus Corporation). The invasion assay was similar to the migration assay except that the Transwell insert was coated with Matrigel. The number of migratory and invasive cells were determined in five randomly selected fields at x200 magnification.

Cell cycle and apoptosis analyses. For the cell cycle assay, cells were seeded in 6-well plates at a density of $2 \times 10^{5}$ cells/ dish. Following incubation at $37^{\circ} \mathrm{C}$ for $48 \mathrm{~h}$, cells were collected, mixed with $70 \%$ ice-cold ethanol and stored overnight at $4^{\circ} \mathrm{C}$. DNA of the cells was then stained with propidium iodide (PI)/RNase staining buffer $(0.5 \mathrm{mg} / \mathrm{ml} \mathrm{PI}$ and $1 \mathrm{mg} / \mathrm{ml}$ RNase A; BD Biosciences, Franklin Lakes, NJ, USA). Following incubation at $37^{\circ} \mathrm{C}$ for $30 \mathrm{~min}$ in the dark, the cells were analyzed using a FACSCalibur flow cytometer (BD Biosciences). To investigate the effect on apoptosis, cells were seeded in 6 -well plates at a density of $2 \times 10^{5}$ cells/dish. Following incubation at $37^{\circ} \mathrm{C}$ for $24 \mathrm{~h}$, cells were treated with gemcitabine (GEM; $2 \mu \mathrm{g} / \mathrm{ml}$ ) or 5 -fluorouracil (5-FU; $50 \mu \mathrm{g} / \mathrm{ml}$ ) for $48 \mathrm{~h}$. PBS served as a negative control. The cells were harvested and stained with Annexin V-fluorescein isothiocyanate/PI (Trevigen, Inc., Gaithersburg, MD, USA) for $15 \mathrm{~min}$ in the dark at room temperature. Finally, the cell populations were analyzed using a FACSCalibur flow cytometer. Cells were treated in triplicate and assayed separately.

Immunofluorescence analyses. Cells were grown on coverslips and fixed with $4 \%$ paraformaldehyde according to the manufacturer's protocol. Following overnight incubation at $4^{\circ} \mathrm{C}$ with mouse anti-epithelial (E-)cadherin antibody (cat. no. ab76055; 1:200 dilution; Abcam, Cambridge, MA, USA), proteins were visualized by incubation with Alexa Fluor 647-conjugated donkey anti-mouse secondary antibody (cat. no. A31571; 
1:50 dilution; Invitrogen; Thermo Fisher Scientific, Inc.). The coverslips were mounted with mounting medium containing DAPI (Prolong Gold Antifade Reagent; Invitrogen; Thermo Fisher Scientific, Inc.). Fluorescence was analyzed using confocal laser microscopy (Olympus Corporation).

Western blotting. Cells were harvested and lysed in radioimmunoprecipitation buffer (Cell Signaling Technology, Inc., Danvers, MA, USA), supplemented with a protease inhibitor cocktail and phenylmethylsulfonyl fluoride (both from Roche Molecular Diagnostics, Pleasanton, CA, USA). The protein concentration was assessed using the Quick Start ${ }^{\mathrm{TM}}$ Bradford protein assay kit (Bio-Rad Laboratories, Inc., Hercules, CA, USA), according to the manufacturer's protocol. Equivalent amounts of proteins $(40 \mu \mathrm{g})$ from each sample were separated by SDS-PAGE (10\% gel) and then transferred onto pre-activated polyvinylidene fluoride membranes (EMD Millipore, Billerica, MA, USA). The blotted membranes were blocked in $5 \%$ fat-free milk in Tris-buffered saline $(20 \mathrm{mM}$ Tris $/ \mathrm{HCl}$, $\mathrm{pH} 7.6,127 \mathrm{mM} \mathrm{NaCl}$ and $0.1 \%$ Tween-20) for $1 \mathrm{~h}$ at room temperature, and incubated with polyclonal primary antibodies against E-cadherin (cat. no. ab40772), neuronal (N-)cadherin (cat. no. ab76011), vimentin (cat. no. ab92547), Slug (cat. no. ab183760); zinc finger E-box-binding homeobox 1 (ZEB1; cat. no. ab203829) and GAPDH (cat. no. ab9485) (all from Abcam) (all at 1:1,000 dilution) at $4^{\circ} \mathrm{C}$ overnight, then with the goat anti-rabbit IgG secondary antibody (cat. no. 7074; 1:2,000 dilution; Cell Signaling Technology, Inc.) at room temperature for $1.5 \mathrm{~h}$. The specific bands were visualized using an enhanced chemiluminescence kit (Pierce; Thermo Fisher Scientific, Inc.) and were subjected to densitometric analysis using AlphaEaseFC software (version 3.1.2; Protein Simple, San Jose, CA, USA). GAPDH was used as a control.

Subcutaneous xenograft transplantation. Female nude mice (BALB/c, nu/nu, $\mathrm{n}=5$ animals per group, four-week-old, 16-18 g) were purchased from the Shanghai Experimental Animal Center (Shanghai, China) and maintained under pathogen-free conditions (temperature, $26-28^{\circ} \mathrm{C}$; air pressure, $0.65 \mathrm{~cm} \mathrm{H}_{2} \mathrm{O}$; relative humidity, 40-60\%; ventilation, 10-15 counts/h; 10-h light/14-h dark cycle; free feed and water intake). All animals were used in accordance with the guidelines of the Institutional Animal Care and Use Committee of the Institute of Nutritional Sciences. Mice were injected subcutaneously into the right posterior flanks with $2 \times 10^{6}$ Panc-1 cells (15). When the tumor reached a volume of $\sim 100 \mathrm{~mm}^{3}$, mice were randomized into the indicated groups and subjected to treatment with 5-FU $(5 \mathrm{mg} / \mathrm{kg})$ intraperitoneally, MK2206 (20 mg/kg) by gastrogavage or PD98059 $(10 \mathrm{mg} / \mathrm{kg})$ by gastrogavage three times weekly. Tumor growth was determined every 5 days for a total of 35 days. At day 35, mice were sacrificed and images of tumors were captured. Tumor volume was determined using Vernier calipers [tumor volume $=$ largest diameter $\mathrm{x}$ (smallest

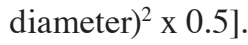

Statistical analysis. Statistical analysis was calculated using SPSS (version 19.0 software; IBM Corp., Armonk, NY, USA) and GraphPad Prism (version 6; GraphPad Software, Inc., La Jolla, CA, USA). Results are presented as the mean \pm standard deviation. Student's t-test and analysis of variance were used to compare quantitative variables. $\chi^{2}$ test and Fisher's exact test were used to compare categorical variables. $\mathrm{P}<0.05$ was considered to indicate a statistically significant difference.

\section{Results}

CUDR is upregulated in PDAC tissues and cell lines. To explore the aberrantly expressed IncRNAs in PDAC, five pairs of human clinical samples were collected, and microarrays were used to analyze differentially expressed lncRNAs and mRNAs between PDAC tissues and matched adjacent non-tumor tissues. A total of 883 lncRNAs and 949 mRNAs were identified that were differentially expressed $(\mathrm{P}<0.05$; data not shown). Hierarchical clustering analysis of these aberrantly expressed lncRNAs and mRNAs exhibited systematic variations between pancreatic cancer tissues and adjacent nontumor tissues (Fig. 1A and B). As a result, the lncRNAs and mRNAs identified were likely to be representative, and these genes that have differential expression possibly participate in the development of pancreatic cancer. Among the differentially expressed IncRNAs, it was observed that lncRNA CUDR was significantly overexpressed in PDAC (fold change 10.6; $\mathrm{P}<0.0001)$.

The CUDR expression level was analyzed using RT-qPCR in a larger clinical sample size of 20 pairs to validate the conclusions of microarray analysis. The result suggested an increased level of CUDR in 16/20 PDAC tissues compared with their matched adjacent normal tissues (median fold change, $2.17 \pm 1.61$; $\mathrm{P}<0.05$; Fig. $1 \mathrm{C}$ ). CUDR expression was investigated in 8 PDAC cell lines and the normal pancreatic ductal epithelial cell line HPDE. Consistent with the results for clinical tissues, the level of CUDR was significantly higher in PDAC cell lines compared with in HPDE cells (Fig. 1D). Thus, these results confirmed that CUDR is upregulated in PDAC tissues and cell lines, suggesting a potential function for CUDR in the development of pancreatic cancer.

CUDR overexpression promotes proliferation by regulating cell cycle progression in PDAC cells. The potential function of CUDR in PDAC cell survival was investigated. According to the different CUDR expression levels in the 8 PDAC cell lines, CUDR was the highest in CFPAC-1 and the lowest in Panc-1 cells. Stable cell lines expressing CUDR was constructed for the relatively lower CUDR-expressing Panc-1 cells (Fig. 2A). An MTT assay indicated that aberrant CUDR expression significantly promoted the proliferation of Panc-1 cells (Fig. 2B). To confirm the effect of CUDR in Panc-1 cells, the expression of endogenous CUDR was stably knocked down using virus-mediated RNAi in the relatively highly expressed CFPAC-1 cells (shCUDR-1 and shCUDR-2; Fig. 2C). The MTT assay also indicated that CUDR depletion significantly decreased the survival of CFPAC-1 cells, whereas shCUDR-2 was more efficient compared with shCUDR-1 (Fig. 2D).

To determine whetherCUDR affects cell cycle progression, cell cycle analysis was performed using fluorescence-activated cell sorting. The results indicated that the proportion of cells in $\mathrm{G}_{2} / \mathrm{M}$ phase was increased in CUDR-overexpressing Panc-1 cells $(\mathrm{P}<0.001)$, and the corresponding proportion of cells in $\mathrm{G}_{0} / \mathrm{G}_{1}$ phase was decreased $(\mathrm{P}<0.001)$ (Fig. $2 \mathrm{E}$ ). Compared with the control group, knocking down CUDR in 

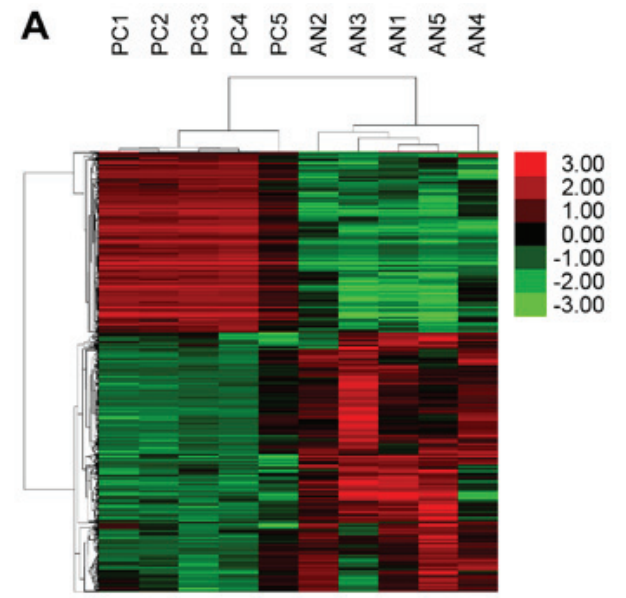

C

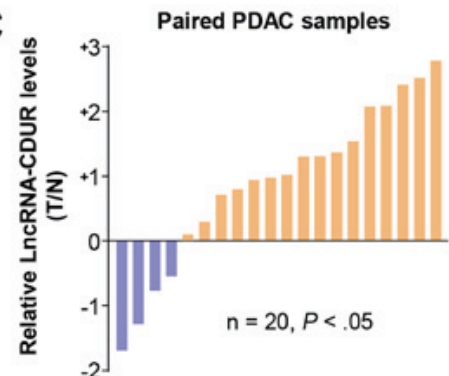

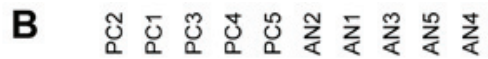
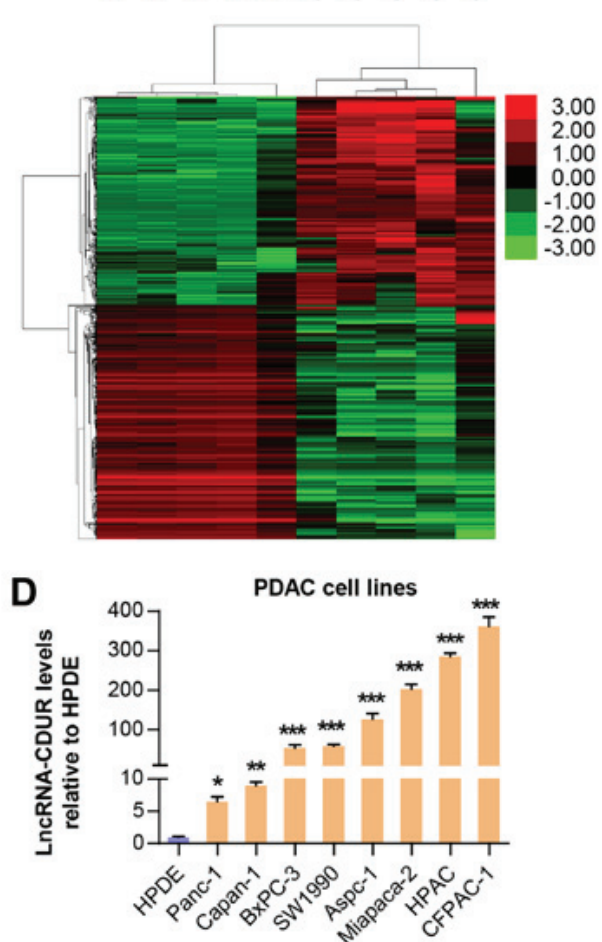

Figure 1. Reverse transcription-quantitative polymerase chain reaction analysis of CUDR in PDAC tissues and cell lines. (A) Hierarchical clustering analysis of aberrantly expressed mRNAs between pancreatic cancer tissues and adjacent non-tumor tissues based on the five pairs of microarrays. (B) Hierarchical clustering analysis of aberrantly expressed long non-coding RNAs between pancreatic cancer tissues and adjacent non-tumor tissues based on the five pairs of microarrays. (C) Relative expression of CUDR in 20 paired human pancreatic cancer tissues and matched adjacent non-tumor tissues. CUDR expression from all tissues was normalized to GAPDH expression $(\Delta \mathrm{Cq})$ and then compared with a non-tumorous tissue and converted into the fold change $\left(2^{-\Delta \Delta C q}\right)$. The median fold change was $2.17 \pm 1.61(\mathrm{P}<0.05)$. Relative CUDR expression levels were determined as $\log _{2}(\mathrm{~T} / \mathrm{N})$. The symbols + and - indicate that the expression is higher or lower, respectively, in the tumor sample compared with in their respective adjacent control tissues. (D) Relative expression of CUDR in 8 PDAC cell lines (Canpan-1, Aspc-1, Panc-1, SW1990, CFPAC-1, BxPC-3, Miapaca-2 and HPAC) and the normal pancreatic ductal epithelial cell line HPDE. The results are presented as the mean \pm standard deviation $(n=3) .{ }^{*} \mathrm{P}<0.05,{ }^{* *} \mathrm{P}<0.01,{ }^{* * * *} \mathrm{P}<0.001$. CUDR, cancer upregulated drug resistance; PDAC, pancreatic ductal adenocarcinoma; $\mathrm{T}$, tumor tissue; $\mathrm{N}$, match adjacent non-tumor tissue.

CFPAC-1 cells led to a significantly decreased proportion of cells in $\mathrm{G}_{2} / \mathrm{M}$ phase $(\mathrm{P}<0.001)$ and an increased proportion of cells in $\mathrm{G}_{0} / \mathrm{G}_{1}$ phase $(\mathrm{P}<0.01)$ (Fig. $\left.2 \mathrm{~F}\right)$. Taken together, these results suggested that CUDR is sufficient to induce a more marked proliferative activity via accelerating the transition of $\mathrm{G}_{0} / \mathrm{G}_{1}$ to $\mathrm{S}-\mathrm{G}_{2} / \mathrm{M}$, thus promoting cell cycle progression in PDAC cells.

CUDR overexpression promotes pancreatic cancer cell migration and invasion in vitro. An increased ability of cell migration and invasion leads to cancer progression and poor prognosis. Therefore, wound-healing and Transwell chamber assays to investigate the effects of CUDR in regulating PDAC cell migration and invasion. The wound-healing assay revealed that ectopic expression of CUDR in Panc-1 cells significantly increased cell motility compared with cells transfected with empty vectors (Fig. 3A), whereas CUDR knockdown in CFPAC-1 cells significantly decreased cell motility compared with the controls (Fig. 3B). Consistently, the Transwell assay also revealed that the ectopic expression of CUDR significantly increased Panc-1 cell migration and invasion (Fig. 3C), and CUDR knockdown significantly decreased CFPAC-1 cell migration and invasion (Fig. 3D). These results demonstrated the critical function of CUDR in promoting PDAC cell migration and invasion.
CUDR overexpression inhibits cell apoptosis and promotes drug resistance in PDAC. In addition to proliferation, increased survival could also be due to changes in apoptosis. To determine whether CUDR influences PDAC cell apoptosis, flow cytometric analysis was performed and it was identified that CUDR overexpression had a marked effect on inhibiting apoptosis in Panc-1 cells (Fig. 4A), whereas CUDR knockdown promoted apoptosis in CFPAC-1 cells (Fig. 4B). To verify whether CUDR had any effect on PDAC cell sensitivity to traditional chemotherapy drugs, PDAC cells were treated with $2 \mu \mathrm{g} / \mathrm{ml}$ GEM or $50 \mu \mathrm{g} / \mathrm{ml} \mathrm{5-FU}$ for $48 \mathrm{~h}$, and the percentage of apoptotic cells was determined. GEM and 5-FU are chemotherapy agents traditionally used to treat PDAC. As presented in Fig. 4A, the proportion of apoptotic cells was decreased in CUDRoverexpressing Panc-1 cells when treated with GEM or 5-FU. Conversely, when CUDR was knocked down by shCUDR-1 or shCUDR-2, the proportion of apoptotic cells was increased in CFPAC-1 cells treated with GEM or 5-FU (Fig. 4B). Furthermore, the CUDR-overexpressing Panc-1 cells exhibited less sensitivity to the chemotherapy agents in the MTT assay, whereas the CUDR-knockdown CFPAC-1 cells exhibited more sensitivity (Fig. 4C) compared with the controls. When treated with 5-FU, the CUDR-overexpressing Panc-1 cells also exhibited less sensitivity in restricting cell migration, compared with the controls (Fig. 4D and E). 

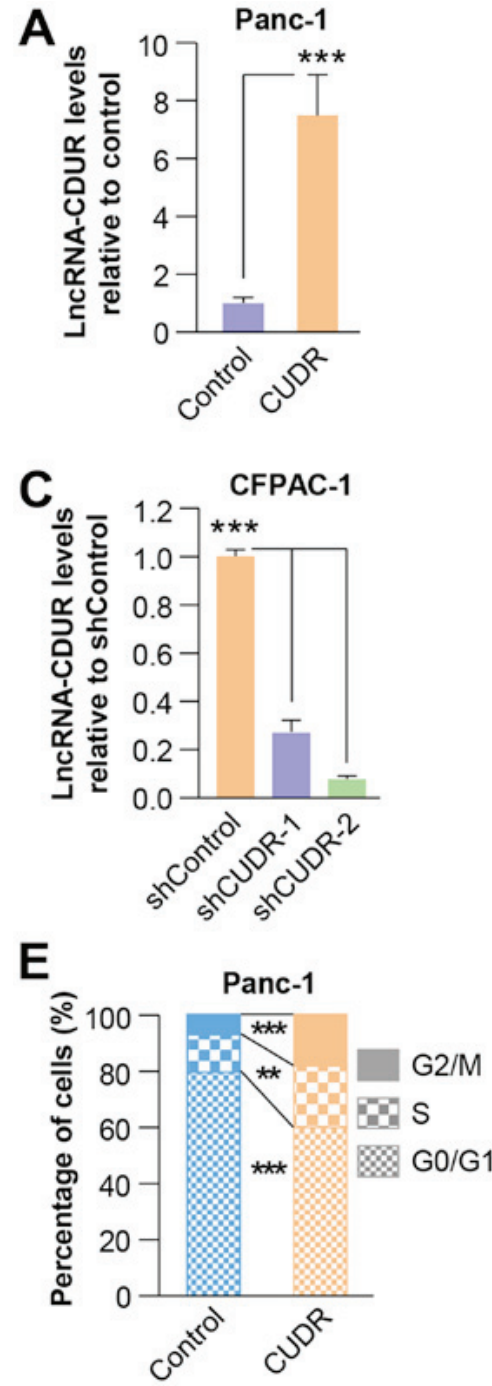
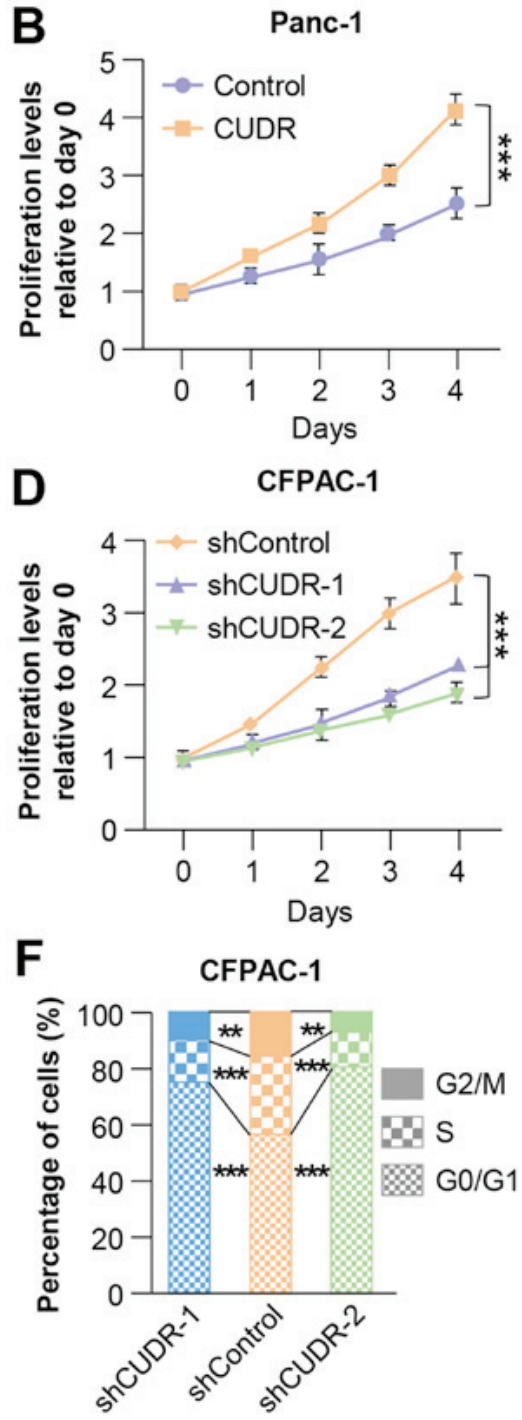

Figure 2. CUDR overexpression promotes proliferation by regulating cell cycle progression. (A) Overexpression of CUDR in the Panc-1 cell line. (B) MTT proliferation assay of Panc-1 cells. (C) Knockdown of CUDR in the CFPAC-1 cell line. (D) MTT proliferation assay of CFPAC-1 cells. Cell cycle analysis of (E) Panc-1 and (F) CFPAC-1 cells. The proportion of cells at various phases $\left(\mathrm{G}_{0} / \mathrm{G}_{1}, \mathrm{~S}\right.$ and $\left.\mathrm{G}_{2} / \mathrm{M}\right)$ was compared between CUDR-overexpressing and CUDR-knocked down cell lines. Results are presented as the mean \pm standard deviation $(n=3)$. ${ }^{* *} \mathrm{P}<0.01,{ }^{* * *} \mathrm{P}<0.001$. CUDR, cancer upregulated drug resistance; sh, short hairpin RNA; lncRNA, long non-coding RNA.

Immunodeficient mice xenografted with CUDRoverexpressing Panc-1 cells or controls were investigated. At 10 days after injection of Panc-1 cells, palpable tumors were present in all mice, and cohorts of mice were treated with 5-FU or PBS. As presented in Fig. $4 \mathrm{~F}$ and G, treatment of mice with 5-FU resulted in limited growth inhibition on the cohorts of CUDR-overexpressing Panc-1 tumors. In contrast, it elicited significant growth inhibition of tumors of the control group. Taken together, these results indicated that CUDR inhibits apoptosis, and induces the chemotherapy resistance of GEM and 5-FU in PDAC cells in vitro and in vivo. In addition, the growth of CUDR-overexpressing Panc-1 tumors was also significantly increased compared with controls treated with PBS. This result indicated a potent growth promotion on CUDR-overexpressing Panc-1 tumors, which is in agreement with results from in vitro assays.

CUDR overexpression promotes PDAC cell epithelialmesenchymal transition (EMT) in vitro. As EMT is one of the crucial mechanisms in cancer metastasis and chemotherapy resistance, it was further investigated whether CUDR induces EMT in PDAC cells. The expression of the epithelial marker E-cadherin was investigated in the constructed PDAC cell lines via immunofluorescence staining. The results revealed that CUDR overexpression decreased E-cadherin expression from the Panc-1 cell membrane (Fig. 5A), whereas CUDR depletion in CFPAC-1 cells led to the opposite effect (Fig. 5B). To support this result, total mRNAs and proteins were extracted from constructed stable Panc-1 and CFPAC-1 cell lines, and the expression of EMT-associated proteins was analyzed using qPCR and western blotting. As presented in Fig. 5C and D, Panc-1 cells with overexpressed CUDR exhibited increased levels of N-cadherin and vimentin, and a decreased level of E-cadherin. CUDR also upregulated the expression level of the EMT-inducing transcription factors Slug and ZEB1. In contrast, CFPAC-1 cells with depleted CUDR levels exhibited a decreased level of N-cadherin, vimentin, Slug and ZEB1, whereas E-cadherin expression was markedly 
A
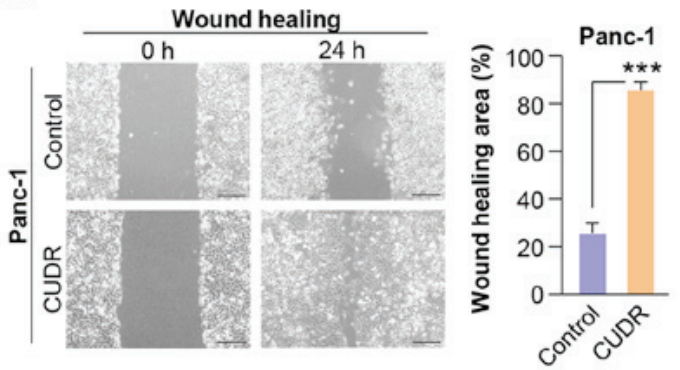

C

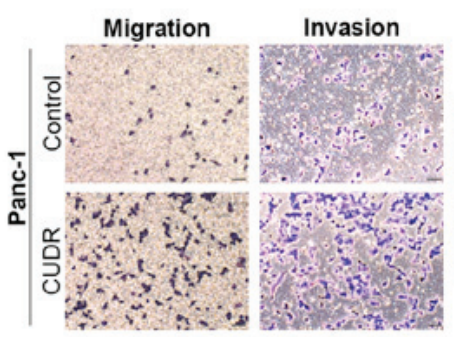

B
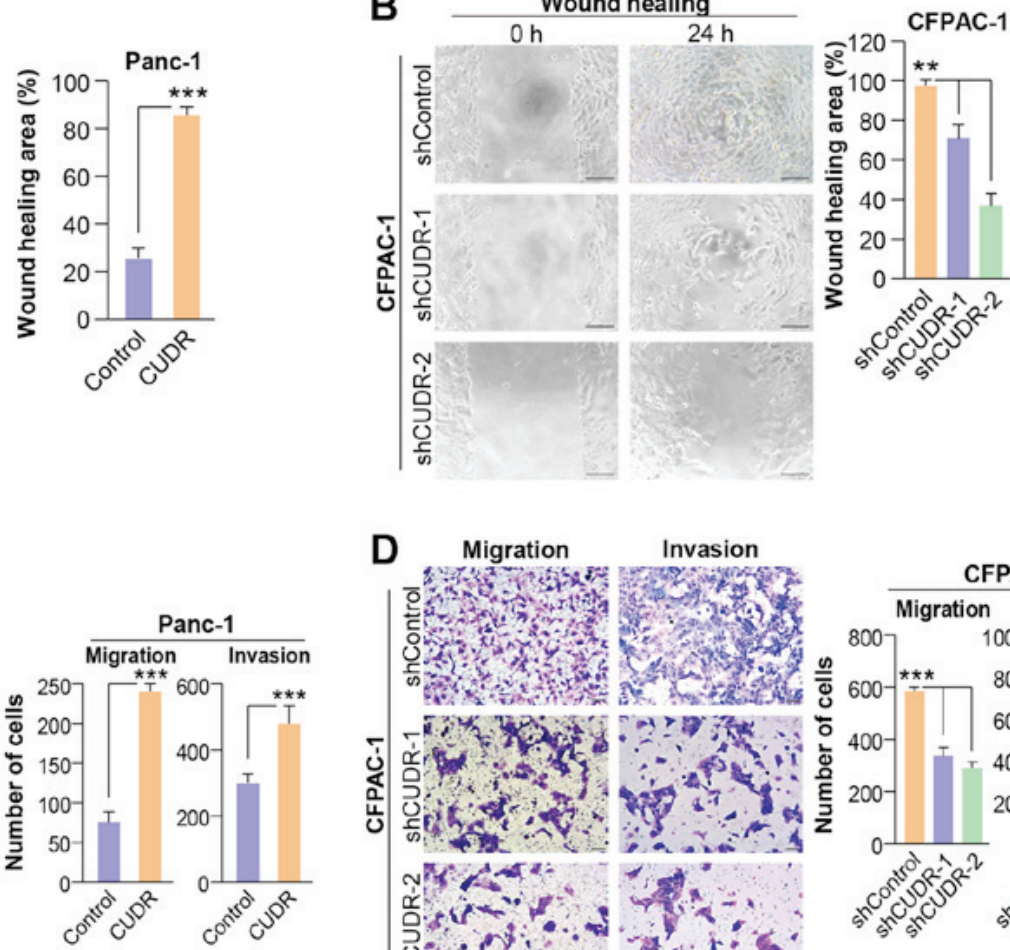

D
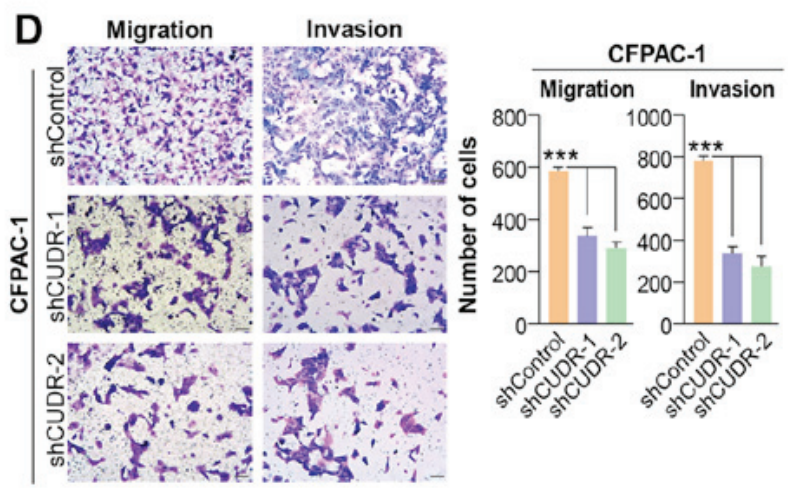

Figure 3. CUDR overexpression promotes pancreatic cancer cell migration and invasion in vitro. Effects of CUDR on the migration of (A) Panc-1 and (B) CFPAC-1 cells was estimated using a wound-healing assay. Effects of CUDR on the migration and invasion of (C) Panc-1 and (D) CFPAC-1 cells was estimated using a Transwell assay. Results are presented as the mean \pm standard deviation $(\mathrm{n}=3)$. ${ }^{* *} \mathrm{P}<0.01,{ }^{* * *} \mathrm{P}<0.001$. Scale bar, $100 \mu \mathrm{m}$. CUDR, cancer upregulated drug resistance; sh, short hairpin RNA.
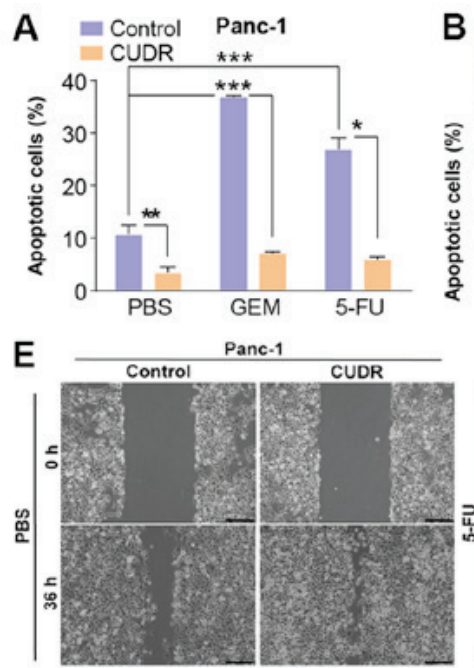
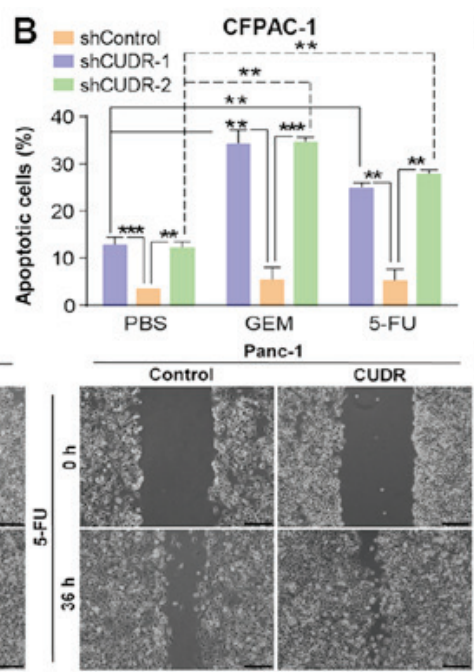

C

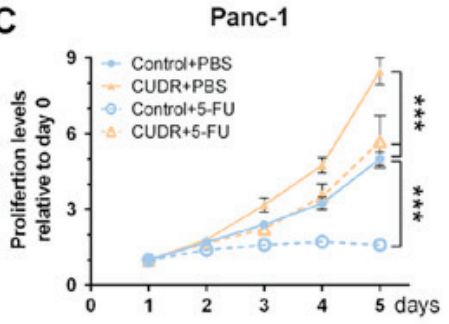

$\mathbf{F}$

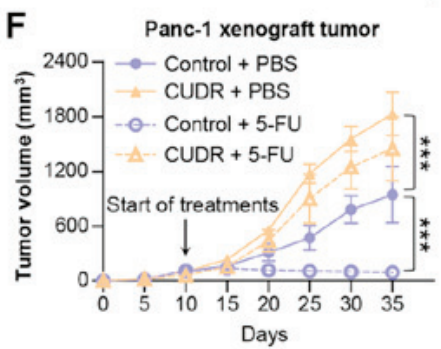

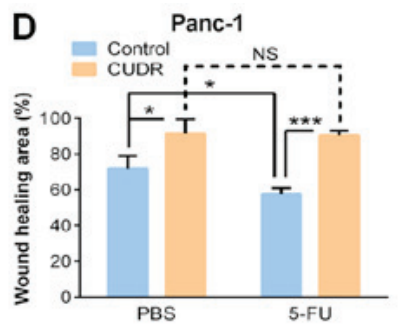

G

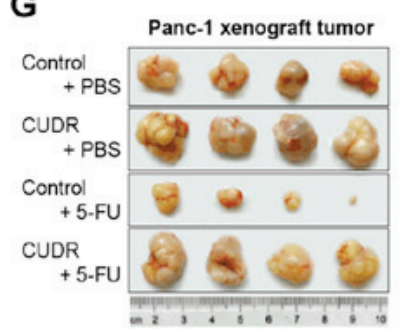

Figure 4. CUDR overexpression inhibits cell apoptosis and promotes drug resistance. (A) Apoptosis analysis of CUDR-overexpressing and control Panc-1 cells and following treatment with $2 \mu \mathrm{g} / \mathrm{ml} \mathrm{GEM}$ or $50 \mu \mathrm{g} / \mathrm{ml} 5$-FU for $48 \mathrm{~h}$. (B) Apoptosis analysis of CUDR-knocked down and control CFPAC-1 cells following treatment with $2 \mu \mathrm{g} / \mathrm{ml} \mathrm{GEM}$ or $50 \mu \mathrm{g} / \mathrm{ml} 5$-FU for $48 \mathrm{~h}$. (C) MTT proliferation assay of CUDR-overexpressing and control Panc-1 cells following treatment with $50 \mu \mathrm{g} / \mathrm{ml} \mathrm{5-FU}$ for $24 \mathrm{~h}$ (D) Quantification and (E) images of wound-healing assay of CUDR-overexpressing and control Panc-1 cells following treatment with $50 \mu \mathrm{g} / \mathrm{ml} 5$-FU for $24 \mathrm{~h}$. The results are presented as the mean \pm standard deviation $(\mathrm{n}=3)$. ${ }^{*} \mathrm{P}<0.05,{ }^{* *} \mathrm{P}<0.01,{ }^{* * * *} \mathrm{P}<0.001$. (F) Quantification and $(\mathrm{G})$ images of in vivo xenograft tumor formation assays were performed using Panc-1 cells $\left(2 \times 10^{6}\right)$ stably transfected with CUDR cDNA or an empty vector, subcutaneously injected into the right posterior flanks of 4-week-old female BALB/c nude mice, followed by treatment with 5-FU (5 mg/kg) or PBS three times weekly when the tumor reached a volume of $\sim 100 \mathrm{~mm}^{3}$ in size. Tumor growth was determined every 5 days. At day 35 , mice were sacrificed and images of tumors were captured. Results are presented as the mean \pm standard deviation $(\mathrm{n}=5)$. ${ }^{*} \mathrm{P}<0.05,{ }^{* *} \mathrm{P}<0.01,{ }^{* * * *} \mathrm{P}<0.001$. Scale bar, $100 \mu \mathrm{m}$. CUDR, cancer upregulated drug resistance; GEM, gemcitabine; 5-FU, 5-fluorouracil; sh, short hairpin.

increased (Fig. 5E and F). These results suggested that CUDR overexpression promotes EMT in PDAC cells.
CUDR regulates PDAC development via activating protein kinase B (AKT) andextracellular-signal-regulated kinase (ERK) 

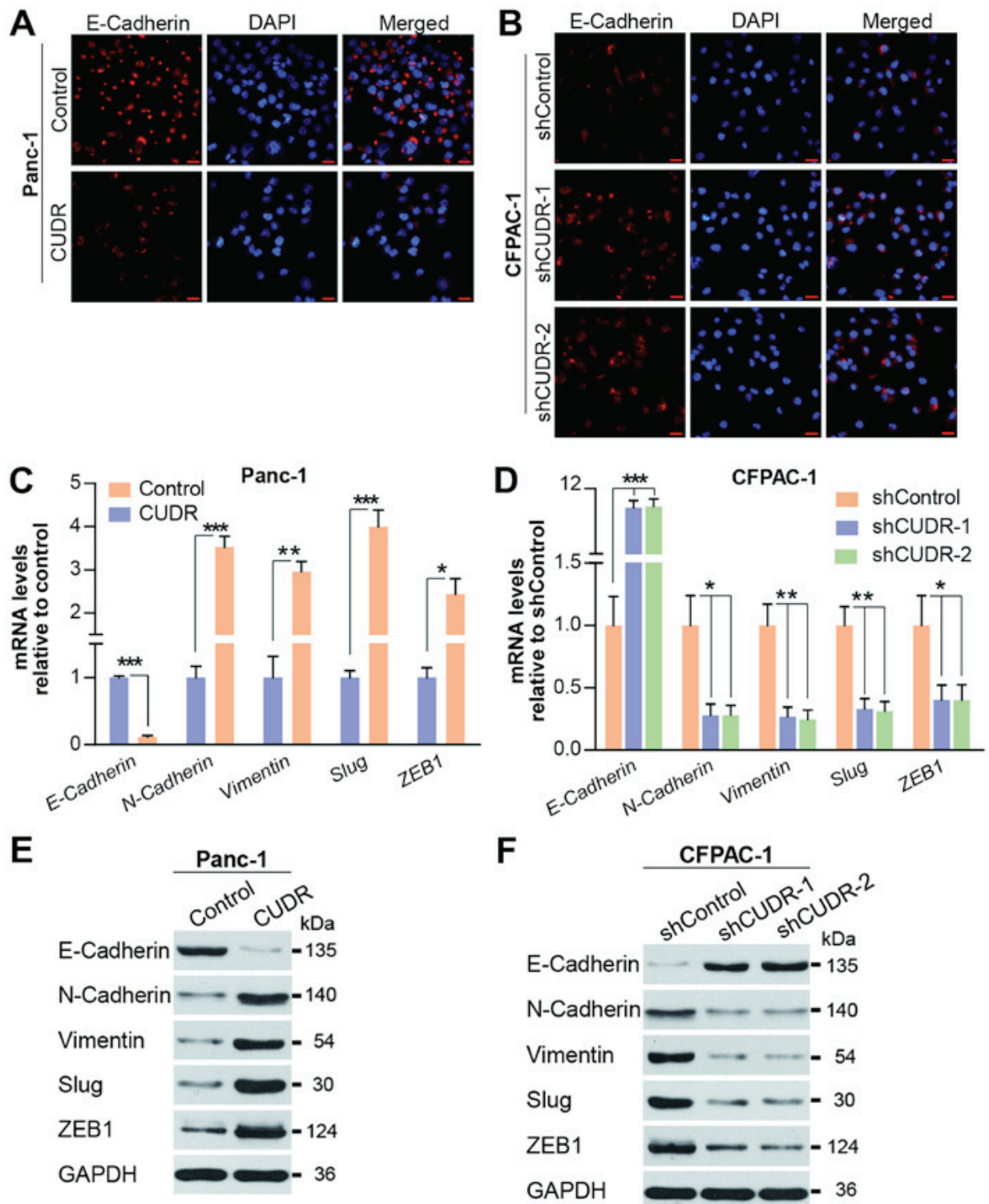

Figure 5. Effect of CUDR in regulating EMT progression. (A) Immunofluorescence images with staining for the epithelial marker E-cadherin for CUDRoverexpressing and control Panc-1 cells. (B) Immunofluorescence images with staining for the epithelial marker E-cadherin of CUDR-knockdown and control CFPAC-1 cells. (C) RT-qPCR analysis of E-cadherin, N-cadherin, vimentin, Slug and ZEB1 expression in CUDR-overexpressing Panc-1 cells compared with the cells transfected with empty vectors. (D) RT-qPCR analysis of E-cadherin, N-cadherin, vimentin, Slug and ZEB1 expression in CFPAC-1 cells transfected with CUDR shRNAs (shCUDR-1 and shCUDR-2) compared with controls. (E) Western blot analysis of E-cadherin, N-cadherin, vimentin, Slug and ZEB1 expression in CUDR-overexpressing Panc-1 cells compared with the cells transfected with empty vectors. (F) Western blot analysis of E-cadherin, $\mathrm{N}$-cadherin, vimentin, Slug and ZEB1 expression in CFPAC-1 cells transfected with CUDR shRNAs (shCUDR-1 and shCUDR-2) compared with controls. The expression of E-cadherin, N-cadherin, vimentin, Slug and ZEB1 was normalized to that of GAPDH. Results are presented as the mean \pm standard deviation (n=3). ${ }^{*} \mathrm{P}<0.05,{ }^{* *} \mathrm{P}<0.01,{ }^{* * *} \mathrm{P}<0.001$. Scale bar, $50 \mu \mathrm{m}$. CUDR, cancer upregulated drug resistance; E-cadherin, epithelial cadherin; RT-qPCR, reverse transcriptionquantitative polymerase chain reaction; N-cadherin, neuronal cadherin; ZEB1, zinc finger E-box-binding homeobox 1; shRNA/sh, short hairpin RNA.

pathways. The underlying molecular mechanisms of the effect of CUDR on PDAC were investigated. Using western blot analysis, it was identified that CUDR overexpression in Panc-1 cells significantly increased phosphorylated (p-) focal adhesion kinase (FAK) and p-AKT levels, whereas the total FAK and AKT were not altered compared with in Panc-1 cells transfected with an empty vector. Reciprocally, CUDR knockdown significantly decreased p-FAK and p-AKT expression, whereas total AKT and FAK levels were not altered in CFPAC-1 cells. p-FAK and p-AKT levels were decreased in the CFPAC-1 cells stably transfected with CUDR-siRNA plasmids with unchanged levels of total FAK and AKT compared with the controls. It was also observed that ERK phosphorylation was markedly increased with unchanged levels of total ERK in CUDR-overexpressed Panc-1 cells and was markedly decreased when CUDR expression was knocked down in CFPAC-1 cells (Fig. 6A). These data suggested that ectopic CUDR expression activates the AKT and ERK signaling pathways by activating their phosphorylation, possibly leading to the promotion of PDAC development.

As the AKT and ERK signaling pathways serve important functions in regulating proliferation, migration and invasion, and promoting EMT progression, it was investigated whether CUDR regulated PDAC cell development via the AKT and ERK signaling pathways. Thus, CUDR-overexpressing Panc-1 cells were treated with MK2206 $(2 \mu \mathrm{M})$, a specific AKT-activation inhibitor, for $24 \mathrm{~h}$ and the MTT assay, flow 
A

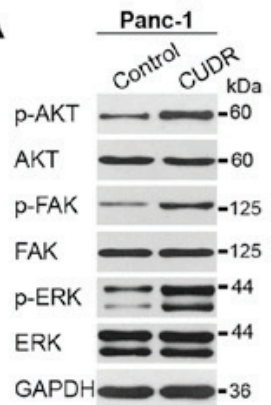

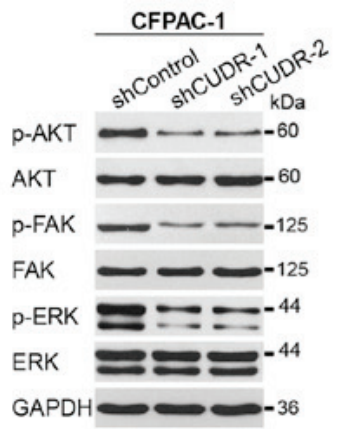

GAPDH
B

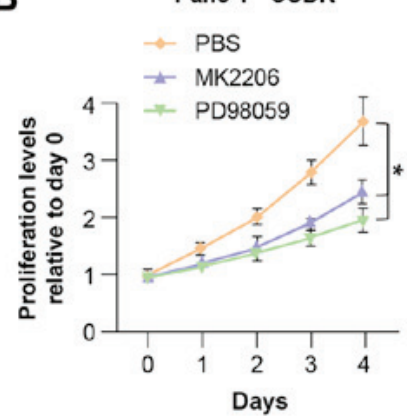

E

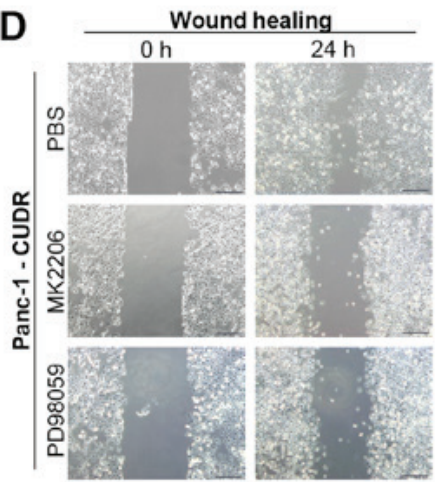

$\mathbf{F}$

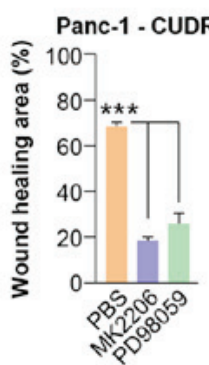

Panc-1
Panc-1

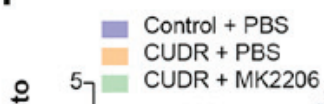

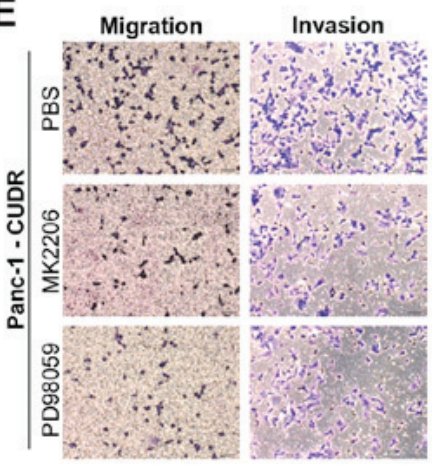

G

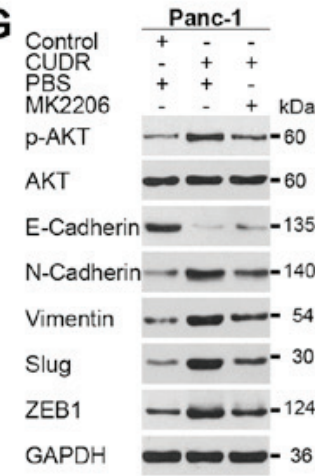

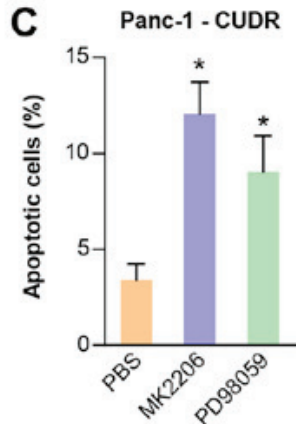
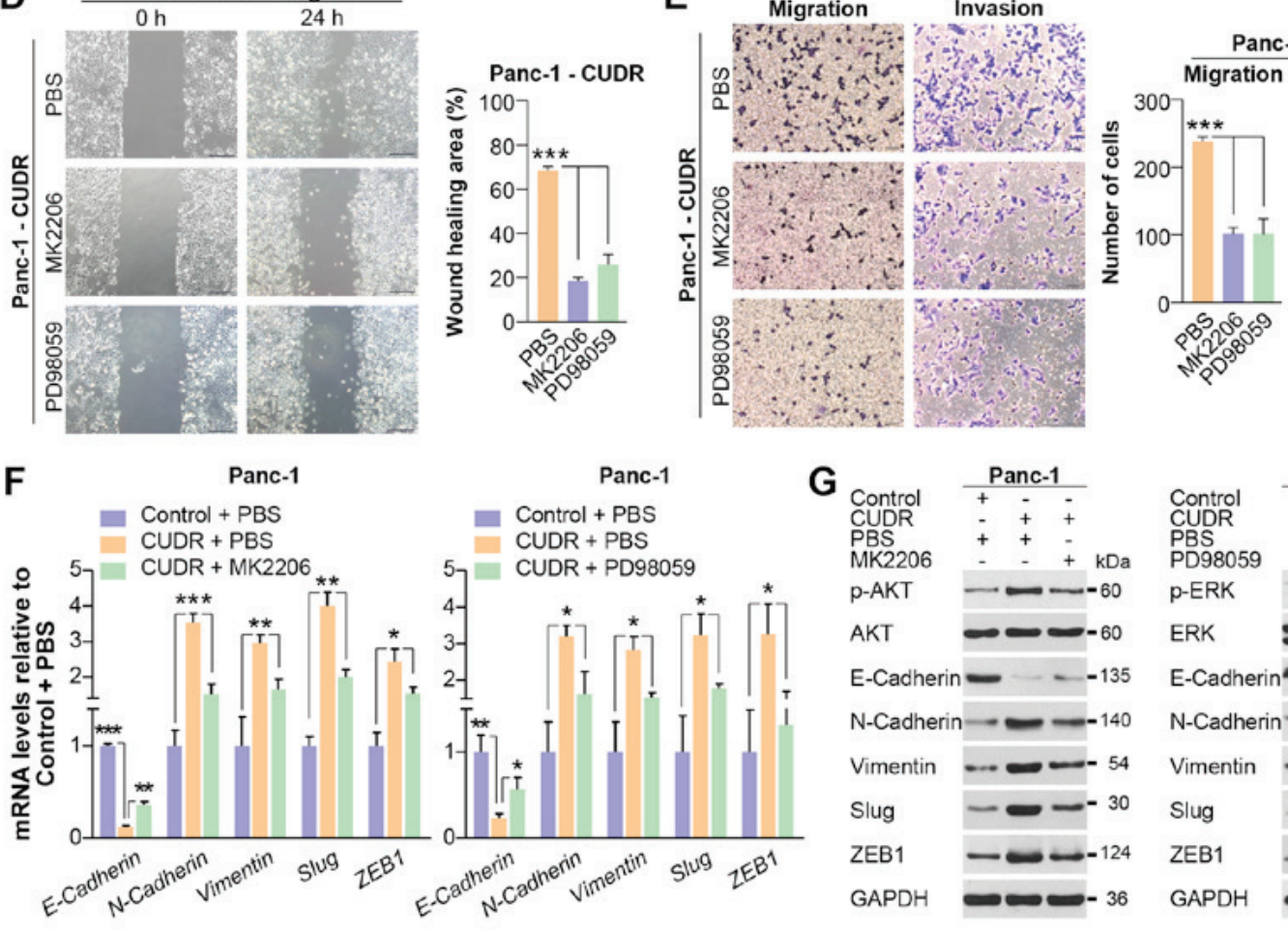

Panc-1 - CUDR
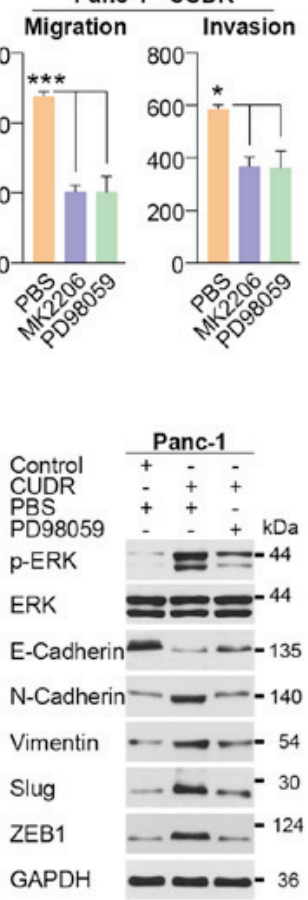

H
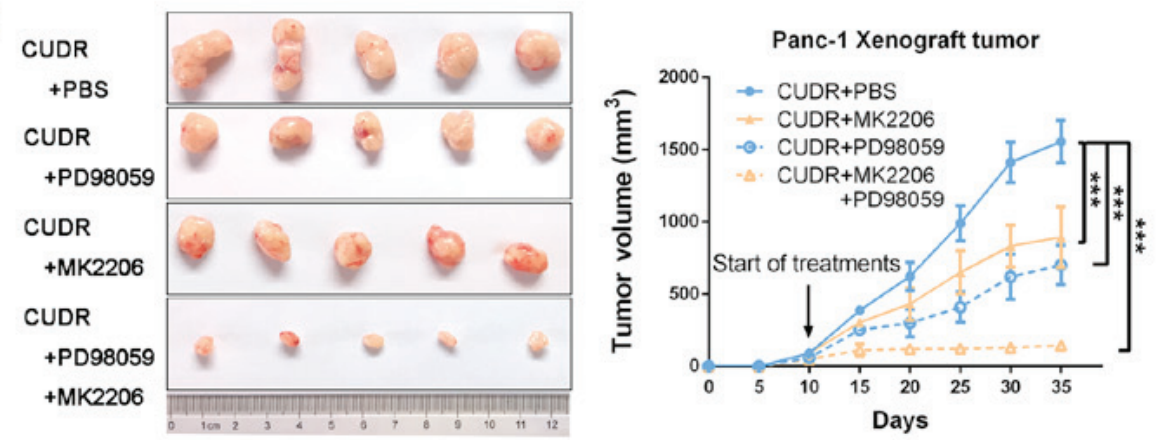

Figure 6. CUDR regulates pancreatic ductal adenocarcinoma development via activating AKT and ERK pathways. (A) Western blot analysis of p-AKT, total AKT, p-FAK, total FAK, p-ERK and total ERK expression in Panc-1 and CFPAC-1 cells stably transfected with CUDR cDNA or CUDR shRNAs (shCUDR-1 and shCUDR-2) compared with controls. The expression levels of p-AKT, total AKT, p-FAK, total FAK, p-ERK and total ERK were normalized to that of GAPDH. (B) MTT proliferation assay of CUDR-overexpressing Panc-1 cells following treatment with MK2206 (2 $\mu \mathrm{M})$ for $24 \mathrm{~h}$, or with PD98059 (10 $\mu \mathrm{M})$ for $48 \mathrm{~h}$ compared with controls. (C) Apoptosis analysis of overexpressing CUDR Panc-1 following treatment with MK2206 (2 $\mu \mathrm{M})$ for $24 \mathrm{~h}$, or with PD98059 (10 $\mu \mathrm{M})$ for $48 \mathrm{~h}$ compared with controls. (D) Wound-healing assay of overexpressing CUDR Panc-1 cells following treatment with MK2206 (2 $\mu \mathrm{M})$ for $24 \mathrm{~h}$, or with PD98059 $(10 \mu \mathrm{M})$ for $48 \mathrm{~h}$ compared with controls. (E) Migration and invasion Transwell assays of CUDR-overexpressing Panc-1 cells following treatment with MK2206 $(2 \mu \mathrm{M})$ for $24 \mathrm{~h}$, or with PD98059 $(10 \mu \mathrm{M})$ for $48 \mathrm{~h}$ compared with controls. (F) Reverse transcription-quantitative polymerase chain reaction analysis and $(\mathrm{G})$ western blot analysis of p-AKT, total AKT, E-cadherin, N-cadherin, vimentin, Slug and ZEB1 expression in Panc-1 cells following treatment with MK2206 $(2 \mu \mathrm{M})$ for $24 \mathrm{~h}$, and p-ERK, total ERK, E-cadherin, N-cadherin, vimentin, Slug and ZEB1 expression in Panc-1 cells following treatment with PD98059 $(10 \mu \mathrm{M})$ for $48 \mathrm{~h}$. The expression of total AKT, p-AKT, total ERK, p-ERK, N-cadherin, vimentin, E-cadherin, Slug and ZEB1 was normalized to that of GAPDH. (H) In vivo xenograft tumor size formation assays were performed using Panc-1 cells (2x106) stably transfected with CUDR cDNA, subcutaneously injected into the right posterior flanks of 4-week-old female BALB/c nude mice, followed by treatment with MK2206 (20 mg/kg) or PD98059 $(10 \mathrm{mg} / \mathrm{kg})$ administered by gastrogavage three times weekly when the tumor reached a volume of $\sim 100 \mathrm{~mm} 3$. Tumor growth was determined every 5 days. At day 35 , mice were sacrificed and images of tumors were captured. Results are presented as the mean \pm standard deviation $(\mathrm{n}=3)$. $* \mathrm{P}<0.05, * * \mathrm{P}<0.01$, ***P $<0.001$. Scale bar, $100 \mu \mathrm{m}$. CUDR, cancer upregulated drug resistance; AKT, protein kinase B; ERK, extracellular-signal-regulated kinase; p-, phosphorylated; shRNA/ sh, short interfering RNA; E-cadherin, epithelial cadherin; N-cadherin, neuronal cadherin; ZEB1, zinc finger E-box-binding homeobox 1. 
cytometric analysis, would-healing and Transwell assays were repeated. The results indicated that CUDR-induced PDAC cell proliferation, migration, invasion and EMT progression were all markedly decreased, and apoptosis was significantly increased in Panc-1 cells treated with MK2206 (Fig. 6B-G). Following treatment of CUDR-overexpressing Panc-1 cells with PD98059 $(10 \mu \mathrm{M})$, a specific ERK-activation inhibitor, for $48 \mathrm{~h}$, CUDR-induced proliferation, migration, invasion and EMT progression were decreased, and CUDR-induced apoptosis was increased (Fig. 6B-G). Together, these results indicated that CUDR regulated PDAC cell proliferation, migration, invasion, apoptosis and EMT progression via the AKT and ERK signaling pathways.

Immunodeficient mice xenografted with CUDRoverexpressing Panc-1 cells or controls were used to investigate these results. At 10 days after injection of Panc-1 cells, palpable tumors were present in all mice, and cohorts of mice were treated with MK2206, PD98059 or PBS. As presented in Fig. 6H, treatment of mice with either MK2206 or PD98059 resulted in marginal growth inhibition on the cohorts of CUDR-overexpressing Panc-1 tumors, and this inhibition was more obvious when treated with the two agents. It suggested that the growth promotion of CUDR-overexpressing Panc-1 tumors is via the AKT and ERK signaling pathways, which was consistent with the results for the in vitro assays.

\section{Discussion}

The lncRNA CUDR, also known as urothelial cancer-associated 1, was identified to be overexpressed in various neoplasms, and it serves key functions in numerous biological progresses. CUDR is upregulated in bladder cancer and promotes tumorigenesis, progression and cisplatin resistance through different mechanisms, and it also represents a potential marker for the diagnosis and prognosis of bladder cancer $(16,17)$. CUDR also serves as an oncogene in a number of types of human cancer, including gastric cancer, colorectal cancer, tongue squamous cell carcinoma, hepatocarcinoma and breast cancer (18-22). Chen et al (23) identified that CUDR expression in pancreatic cancer is significantly correlated with malignant factors, including tumor size, depth of invasion, cancer antigen 19-9 level and tumor stage. Fu et al (24) identified that CUDR is involved in PDAC progression and is associated with the overall survival of patients with PDAC. Zhang et al (25) suggested that microRNA-135a may be a target of CUDR in regulating the growth and metastasis of pancreatic cancer. Cheng et al (26) indicated that CUDR may activate AKT/ mammalian target of rapamycin and ERK signaling pathways, and EMT in epidermal growth factor receptor-mutant nonsmall cell lung cancer. Wang et al (27) identified that CUDR promotes cell proliferation via recruiting enhancer of Zeste homolog 2 and activating AKT in gastric cancer. However, the further function and underlying molecular mechanism of CUDR in regulating PDAC development and progression remain unknown.

In the present study, microarrays of PDAC tissues and matched adjacent non-tumor tissues were used to profile different lncRNA expression, and 883 lncRNAs and 949 mRNAs were obtained that were differentially expressed. Among these lncRNAs, it was confirmed that CUDR is overexpressed in PDAC tissues and 8 PDAC cell lines, and increases PDAC cell progression, migration and invasion, and inhibits apoptosis; in contrast, CUDR knockdown had the opposite effects, indicating a marked oncogenic function. The results of the present study also indicated that aberrant CUDR expression inhibited PDAC cell apoptosis induced by GEM and 5-FU, whereas CUDR knockdown had the opposite effect. As GEM and 5-FU are traditional chemotherapy drugs for PDAC, these results indicated that CUDR may decrease tumor sensitivity to GEM and 5-FU chemotherapy, inducing drug resistance in patients with pancreatic cancer and contributing to a poor prognosis. In addition, CUDR-induced progression and drug resistance of 5-FU was confirmed in vivo.

As migration and invasion are crucial for metastasis, which is responsible for the mortality of $>90 \%$ of patients with PDAC, the focus of the present study was on the underlying molecular mechanism of how CUDR promotes PDAC cell migration and invasion. Previous study has indicated that EMT serves a pivotal and intricate function in tumor metastasis, and also serves an important function in pancreatic cancer invasion and metastasis (28). To the best of our knowledge, only a limited number of lncRNAs have been associated with EMT in PDAC: LOC389641 (8), lincRNA-ROR (29), MALAT-1 (30), MEG3 (31), HOTAIR (32) and H19 (33). The results of the present study provide the first evidence that CUDR also promotes EMT in PDAC. Ectopic CUDR expression upregulated the expression level of the mesenchymal markers $\mathrm{N}$-cadherin and vimentin, and downregulated the expression level of the epithelial marker E-cadherin. CUDR also upregulated the expression of Slug and ZEB1, which are vital transcription factors for inducing EMT $(29,34)$. Conversely, the loss of CUDR had the opposite effect.

The ectopic expression of CUDR was identified to activate the AKT and ERK signaling pathways via activating FAK, AKT and ERK phosphorylation, whereas CUDR knockdown led to decreased FAK, AKT and ERK activation. FAK is known to control the mesenchymal characteristics imparting adhesion and invasiveness in cancer cells (35), and phosphorylated FAK induces downstream AKT pathway activation (36). Activation of the AKT signaling pathway is correlated with cell cycle progression, migration and invasion, and may decrease cell apoptosis in a number of types of cancer $(35,37)$. In addition, AKT is activated and promotes invasiveness in human pancreatic cancer (38). ERK, as an important member of the mitogen-activated protein kinase signaling pathway, is a crucial kinase in regulating tumorigenesis and progression. Persistent ERK activation regulates cell cycle arrest and promote tumor cell growth, proliferation and differentiation $(39,40)$. Consistent with the results of the present study, when treated with MK2206 and PD98059, the effect of CUDR on cell proliferation, apoptosis, migration and invasion could be inhibited. Thus, we hypothesize that CUDR promotes proliferation, migration and invasion, and inhibits cell apoptosis via activating the AKT and ERK signaling pathways. Furthermore, $90 \%$ of patients with pancreatic cancer harbor oncogenic point mutations in $K R A S$, which lead to constitutive KRAS activation, thus cell lines without KRAS mutations are possibly not representative of the majority of pancreatic cancer types. CFPAC-1 and PANC-1 cells are the most commonly used pancreatic cancer cell lines, and contain KRAS mutations (41). As it is known 
that oncogenic KRAS activates ERK and AKT signaling pathways (42), which are also suggested to be regulated by CUDR in the present study, we hypothesized that CUDR may be associated with KRAS activation. However, no association between CUDR and KRAS was identified in an mRNA array assay (C. Shao, unpublished data), indicating that the KRAS pathway is unlikely to be involved in CUDR downstream. Further experiments are required to dissect the mechanisms by which CUDR activates AKT and ERK signaling pathways.

Activation of the AKT signaling pathway is an important feature of EMT (43). Results of our previous study had also suggested that EMT is initiated by the AKT/Snail signaling pathway in pancreatic cancer, promoting cell metastasis and invasion (44). The ERK signaling pathway has been confirmed to have another vital function in promoting EMT (45). Consistent with the results of the present study, we therefore hypothesize that CUDR overexpression promotes EMT via the activation of AKT and ERK signaling pathways. In support of this hypothesis, in the present study, when AKT phosphorylation was abolished by its specific inhibitor MK2206 or ERK phosphorylation was abolished by its specific inhibitor PD98059 in PDAC cells, CUDR-induced EMT progression was able to be restored. Thus, it is concluded that CUDR promotes EMT via activating the AKT and ERK signaling pathways in PDAC cells. In addition, EMT contributes to drug resistance (46). An inverse association between E-cadherin and ZEB1 was also identified to be associated with resistance to GEM, 5-FU and other chemotherapy drugs in pancreatic cancer $(47,48)$. The results of the present study indicated that CUDR inhibited the apoptosis induced by GEM and 5-FU, promoted the drug resistance of GEM and 5-FU, and promoted EMT in PDAC cells; CUDR also induces the chemotherapy resistance of 5-FU in PDAC cells in vivo. Furthermore, the results of the present study indicated that chemosensitivity to 5-FU in PDAC cells could be decreased by CUDR through promoting EMT. Considering that IncRNAs exhibit versatility in molecular function, it should be noted that promoting EMT and activating FAK/ AKT and ERK signaling pathways are only some of the mechanisms for regulating pancreatic cancer migration, invasion, metastasis and apoptosis. Alternative mechanisms of CUDR in promoting pancreatic cancer progression require investigation.

In conclusion, the present study has identified differentially expressed lncRNAs and demonstrated that CUDR is overexpressed in PDAC. CUDR is able to increase PDAC cell proliferation, migration and invasion, inhibit apoptosis, and promote drug resistance; it also regulates PDAC cell EMT. The CUDR-induced PDAC malignant phenotypes is via the AKT and ERK signaling pathways. Downregulation of CUDR may therefore provide a novel therapeutic strategy to prevent PDAC progression, metastasis and drug resistance in the future.

\section{Acknowledgements}

The authors acknowledge the members of the Key Laboratory of Food Safety Research, particularly Wei Wei, Chen Dai, Le-Le Song, Ren-Xu Chang, Wei-Wei Ji and Yi Xu for excellent technical assistance.

\section{Funding}

The present study was supported by the Science and Technology Commission of Shanghai Municipality (grant no. 124119a0501), Second Military Medical University (grant nos. 2017JZ27 and 2017CZQN03) and the Chinese Academy of Sciences (grant no. XDA12010102).

\section{Availability of data and materials}

All data generated or analyzed during this study are included in this published article. The microarray data were published in the Gene Expression Omnibus database (www.ncbi.nlm. nih.gov/geo) under accession number GSE101094 as part of this study.

\section{Authors' contributions}

XL and MQ performed all the experiments and analyzed the data. RW and LT provided support with experimental materials and techniques. AL, DC, JC and XH provided human specimens. WL analyzed the data. CS and LZ designed the research and revised the manuscript. XL wrote the manuscript. All authors reviewed and approved the final manuscript.

\section{Ethics approval and consent to participate}

The patient study and the animal experiments were approved by the Ethics Committee for Biomedical Research of Second Military Medical University. Written informed consent was obtained from all patients or their relatives.

\section{Patient consent for publication}

Not applicable.

\section{Competing interests}

The authors declare that they have no competing interests.

\section{References}

1. Siegel RL, Miller KD and Jemal A: Cancer statistics, 2018. CA Cancer J Clin 68: 7-30, 2018.

2. Poruk KE, Firpo MA, Adler DG and Mulvihill SJ: Screening for pancreatic cancer: Why, how, and who? Ann Surg 257: 17-26, 2013.

3. Ryan DP, Hong TS and Bardeesy N: Pancreatic adenocarcinoma. N Engl J Med 371: 1039-1049, 2014.

4. Uesaka K, Boku N, Fukutomi A, Okamura Y, Konishi M, Matsumoto I, Kaneoka Y, Shimizu Y, Nakamori S, Sakamoto H, et al; JASPAC 01 Study Group: Adjuvant chemotherapy of S-1 versus gemcitabine for resected pancreatic cancer: A phase 3 , open-label, randomised, non-inferiority trial (JASPAC 01). Lancet 388: 248-257, 2016.

5. Xiong XD, Ren X, Cai MY, Yang JW, Liu X and Yang JM: Long non-coding RNAs: An emerging powerhouse in the battle between life and death of tumor cells. Drug Resist Updat 26: 28-42, 2016.

6. Qi P and Du X: The long non-coding RNAs, a new cancer diagnostic and therapeutic gold mine. Mod Pathol 26: 155-165, 2013.

7. Xie Z, Chen X, Li J, Guo Y, Li H, Pan X, Jiang J, Liu H and Wu B: Salivary HOTAIR and PVT1 as novel biomarkers for early pancreatic cancer. Oncotarget 7: 25408-25419, 2016. 
8. Zheng S, Chen H, Wang Y, Gao W, Fu Z, Zhou Q, Jiang Y, Lin Q, Tan L, Ye H, et al: Long non-coding RNA LOC389641 promotes progression of pancreatic ductal adenocarcinoma and increases cell invasion by regulating E-cadherin in a TNFRSF10A-related manner. Cancer Lett 371: 354-365, 2016.

9. Li X, Deng SJ, Zhu S, Jin Y, Cui SP, Chen JY, Xiang C, Li QY, He C, Zhao SF, et al: Hypoxia-induced lncRNA-NUTF2P3-001 contributes to tumorigenesis of pancreatic cancer by derepressing the miR-3923/KRAS pathway. Oncotarget 7: 6000-6014, 2016.

10. Livak KJ and Schmittgen TD: Analysis of relative gene expression data using real-time quantitative PCR and the 2(-Delta Delta $\mathrm{C}(\mathrm{T})$ ) method. Methods 25: 402-408, 2001.

11. Zhang X, Sun S, Pu JK, Tsang AC, Lee D, Man VO, Lui WM, Wong ST and Leung GK: Long non-coding RNA expression profiles predict clinical phenotypes in glioma. Neurobiol Dis 48: $1-8,2012$.

12. Harrow J, Denoeud F, Frankish A, Reymond A, Chen CK, Chrast J, Lagarde J, Gilbert JG, Storey R, Swarbreck D, et al GENCODE: producing a reference annotation for ENCODE. Genome Biol 7 (Suppl 1): S4.1-9, 2006.

13. Ørom UA, Derrien T, Beringer M, Gumireddy K, Gardini A, Bussotti G, Lai F, Zytnicki M, Notredame C, Huang Q, et al: Long noncoding RNAs with enhancer-like function in human cells. Cell 143: 46-58, 2010

14. Guttman M, Amit I, Garber M, French C, Lin MF, Feldser D, Huarte M, Zuk O, Carey BW, Cassady JP, et al: Chromatin signature reveals over a thousand highly conserved large noncoding RNAs in mammals. Nature 458: 223-227, 2009.

15. Miyake T, Honma Y, Urano T, Kato N and Suzumiya J: Combined treatment with tamoxifen and a fusicoccin derivative (ISIR-042) to overcome resistance to therapy and to enhance the antitumor activity of 5-fluorouracil and gemcitabine in pancreatic cancer cells. Int J Oncol 47: 315-324, 2015.

16. Wang Y, Chen W, Yang C, Wu W, Wu S, Qin X and Li X: Long non-coding RNA UCA1a(CUDR) promotes proliferation and tumorigenesis of bladder cancer. Int J Oncol 41: 276-284, 2012.

17. Tsang WP, Wong TWL, Cheung AHH, Co CNN and Kwok TT: Induction of drug resistance and transformation in human cancer cells by the noncoding RNA CUDR. RNA 13: 890-898, 2007.

18. Huang J, Zhou N, Watabe K, Lu Z, Wu F, Xu M and Mo YY: Long non-coding RNA UCA1 promotes breast tumor growth by suppression of p27 (Kip1). Cell Death Dis 5: e1008, 2014.

19. Pu H, Zheng Q, Li H, Wu M, An J, Gui X, Li T and Lu D: CUDR promotes liver cancer stem cell growth through upregulating TERT and C-Myc. Oncotarget 6: 40775-40798, 2015.

20. Han Y, Yang YN, Yuan HH, Zhang TT, Sui H, Wei XL, Liu L, Huang P, Zhang WJ and Bai YX: UCA1, a long non-coding RNA up-regulated in colorectal cancer influences cell proliferation, apoptosis and cell cycle distribution. Pathology 46: 396-401, 2014.

21. Zheng Q, Wu F, Dai WY, Zheng DC, Zheng C, Ye H, Zhou B, Chen JJ and Chen P: Aberrant expression of UCA1 in gastric cancer and its clinical significance. Clin Transl Oncol 17: 640-646, 2015.

22. Fang Z, Wu L, Wang L, Yang Y, Meng Y and Yang H: Increased expression of the long non-coding RNA UCA1 in tongue squamous cell carcinomas: A possible correlation with cancer metastasis. Oral Surg Oral Med Oral Pathol Oral Radiol 117: 89-95, 2014.

23. Chen P, Wan D, Zheng D, Zheng Q, Wu F and Zhi Q: Long noncoding RNA UCA1 promotes the tumorigenesis in pancreatic cancer. Biomed Pharmacother 83: 1220-1226, 2016.

24. Fu XL, Liu DJ, Yan TT, Yang JY, Yang MW, Li J, Huo YM, Liu W, Zhang JF, Hong J, et al: Analysis of long non-coding RNA expression profiles in pancreatic ductal adenocarcinoma. Sci Rep 6: 33535, 2016.

25. Zhang X, Gao F, Zhou L, Wang H, Shi G and Tan X: UCA1 regulates the growth and metastasis of pancreatic cancer by sponging miR-135a. Oncol Res 25: 1529-1541, 2017.

26. Cheng N, Cai W, Ren S, Li X, Wang Q, Pan H, Zhao M, Li J, Zhang Y, Zhao C, et al: Long non-coding RNA UCA1 induces non-T790M acquired resistance to EGFR-TKIs by activating the AKT/mTOR pathway in EGFR-mutant non-small cell lung cancer. Oncotarget 6: 23582-23593, 2015.

27. Wang ZQ, Cai Q, Hu L, He CY, Li JF, Quan ZW, Liu BY, Li C and Zhu ZG: Long noncoding RNA UCA1 induced by SP1 promotes cell proliferation via recruiting EZH2 and activating AKT pathway in gastric cancer. Cell Death Dis 8: e2839, 2017.

28. Pan JJ and Yang MH: The role of epithelial-mesenchymal transition in pancreatic cancer. J Gastrointest Oncol 2: 151-156, 2011.
29. Zhan HX, Wang Y, Li C, Xu JW, Zhou B, Zhu JK, Han HF, Wang L, Wang YS and Hu SY: LincRNA-ROR promotes invasion, metastasis and tumor growth in pancreatic cancer through activating ZEB1 pathway. Cancer Lett 374: 261-271, 2016.

30. Jiao F, Hu H, Yuan C, Wang L, Jiang W, Jin Z, Guo Z and Wang L: Elevated expression level of long noncoding RNA MALAT-1 facilitates cell growth, migration and invasion in pancreatic cancer. Oncol Rep 32: 2485-2492, 2014.

31. Ma L, Wang F, Du C, Zhang Z, Guo H, Xie X, Gao H, Zhuang Y, Kornmann M, Gao H, et al: Long non-coding RNA MEG3 functions as a tumour suppressor and has prognostic predictive value in human pancreatic cancer. Oncol Rep 39: 1132-1140, 2018.

32. Ren Y, Jia HH, Xu YQ, Zhou X, Zhao XH, Wang YF, Song X, Zhu ZY, Sun T, Dou Y, et al: Paracrine and epigenetic control of CAF-induced metastasis: The role of HOTAIR stimulated by TGF-ß1 secretion. Mol Cancer 17: 5, 2018.

33. Ma C, Nong K, Zhu H, Wang W, Huang X, Yuan Z and Ai K: H19 promotes pancreatic cancer metastasis by derepressing let-7's suppression on its target HMGA2-mediated EMT. Tumour Biol 35: 9163-9169, 2014.

34. Taube JH, Herschkowitz JI, Komurov K, Zhou AY, Gupta S, Yang J, Hartwell K, Onder TT, Gupta PB, Evans KW, et al: Core epithelial-to-mesenchymal transition interactome gene-expression signature is associated with claudin-low and metaplastic breast cancer subtypes. Proc Natl Acad Sci USA 107: 15449-15454, 2010.

35. Thamilselvan V, Craig DH and Basson MD: FAK association with multiple signal proteins mediates pressure-induced colon cancer cell adhesion via a Src-dependent PI3K/Akt pathway. FASEB J 21: 1730-1741, 2007.

36. Miyazaki T, Karube M, Matsuzaki Y, Ikegami T, Doy M, Tanaka $\mathrm{N}$ and Bouscarel $\mathrm{B}$ : Taurine inhibits oxidative damage and prevents fibrosis in carbon tetrachloride-induced hepatic fibrosis. J Hepatol 43: 117-125, 2005

37. Itoh N, Semba S, Ito M, Takeda H, Kawata S and Yamakawa M: Phosphorylation of Akt/PKB is required for suppression of cancer cell apoptosis and tumor progression in human colorectal carcinoma. Cancer 94: 3127-3134, 2002.

38. Tanno S, Tanno S, Mitsuuchi Y, Altomare DA, Xiao GH and Testa JR: AKT activation up-regulates insulin-like growth factor I receptor expression and promotes invasiveness of human pancreatic cancer cells. Cancer Res 61: 589-593, 2001.

39. Tao C, Lin H and Chen S: The regulation of ERK and p-ERK expression by cisplatin and sorafenib in gastric cancer cells. Gene 552: 106-115, 2014.

40. Ohtsuka S, Ogawa S, Wakamatsu E and Abe R: Cell cycle arrest caused by MEK/ERK signaling is a mechanism for suppressing growth of antigen-hyperstimulated effector $\mathrm{T}$ cells. Int Immunol 28: 547-557, 2016

41. Deer EL, González-Hernández J, Coursen JD, Shea JE, Ngatia J, Scaife CL, Firpo MA and Mulvihill SJ: Phenotype and genotype of pancreatic cancer cell lines. Pancreas 39: 425-435, 2010.

42. Mann KM, Ying H, Juan J, Jenkins NA and Copeland NG KRAS-related proteins in pancreatic cancer. Pharmacol Ther 168: 29-42, 2016

43. Larue L and Bellacosa A: Epithelial-mesenchymal transition in development and cancer: Role of phosphatidylinositol 3' kinase/ AKT pathways. Oncogene 24: 7443-7454, 2005.

44. Liu A, Shao C, Jin G, Liu R, Hao J, Song B, Ouyang L and Hu X: miR-208-induced epithelial to mesenchymal transition of pancreatic cancer cells promotes cell metastasis and invasion. Cell Biochem Biophys 69: 341-346, 2014.

45. Tashiro E, Henmi S, Odake H, Ino S and Imoto M: Involvement of the MEK/ERK pathway in EGF-induced E-cadherin downregulation. Biochem Biophys Res Commun 477: 801-806, 2016.

46. Iser IC, Pereira MB, Lenz G and Wink MR: The epithelialto-mesenchymal transition-like process in glioblastoma: An updated systematic review and in silico investigation. Med Res Rev 37: 271-313, 2017.

47. Zheng X, Carstens JL, Kim J, Scheible M, Kaye J, Sugimoto H, Wu CC, LeBleu VS and Kalluri R: Epithelial-to-mesenchymal transition is dispensable for metastasis but induces chemoresistance in pancreatic cancer. Nature 527: 525-530, 2015.

48. Arumugam T, Ramachandran V, Fournier KF, Wang H, Marquis L, Abbruzzese JL, Gallick GE, Logsdon CD, McConkey DJ and Choi W: Epithelial to mesenchymal transition contributes to drug resistance in pancreatic cancer. Cancer Res 69: 5820-5828, 2009. 